> La localisation intracellulaire des ARN est, dans de nombreuses espèces, impliquée dans les phénomènes de polarité cellulaire. Le plus souvent, I'ARN se localise en fonction d'une polarité préexistante et n'influence pas cette asymétrie. Cependant, dans le cas de l'actine $\beta$ des vertébrés, le transport et la localisation de cet ARN sont directement impliqués dans l'établissement et le maintien de la polarisation cellulaire. Cela est vrai dans des cellules fibroblastiques en déplacement, mais aussi lors de la croissance axonale des neurones immatures. De manière remarquable, une altération du transport de cet ARN pourrait être liée à l'apparition de maladies chez l'homme. Cet article décrit l'état actuel des connaissances sur le rôle et les mécanismes de la localisation de l'ARNm de l'actine $\beta$. <

\section{Polarité cellulaire et localisation intracellulaire des ARNm de l'actine}

Brigitte Lavoie, Eugenia Basyuk, Rémy Bordonné, Édouard Bertrand

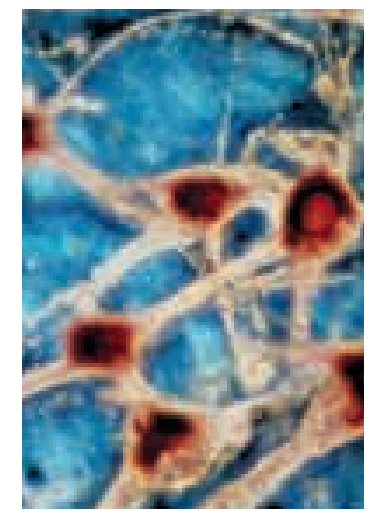

rentes régions de la cellule [4]. Ainsi, les ARNm de l'actine $\alpha$ et $\gamma$ sont présents dans une zone périnucléaire, tandis que celui de l'actine $\beta$ est localisé à proximité des zones riches en actine de la périphérie cellulaire, comme la leading edge des fibroblastes, le cône de croissance des neurones immatures et les épines dendritiques des régions postsynaptiques des neurones matures.

Il est possible de bloquer la localisation de l'ARNm de l'actine $\beta$ dans des cellules vivantes grâce à des oligonucléotides antisens qui peuvent s'apparier in vivo à la séquence de localisation de l'ARN et empêcher sa fonction. Ainsi, il a été démontré que l'inhibition de la localisation de l'ARNm de l'actine $\beta$ entraîne une désorganisation du cytosquelette d'actine dans les fibroblastes, ainsi que l'incapacité de la cellule à se mouvoir continûment dans une même direction [5-7]. Ce phénomène intervient sans que la quantité totale d'actine $\beta$ soit modifée, indiquant que la localisation de l'ARNm participe à la polarisation du cytosquelette. De manière intéressante, le pouvoir invasif des cellules 
cancéreuses augmente avec leur capacité à se déplacer de manière désordonnée, et avec leur incapacité à localiser correctement l'ARNm de l'actine $\beta$ [8].

Lorsque les fibroblastes se déplacent, l'actine de la leading edge est soumise à des cycles rapides de polymérisation/dépolymérisation, producteurs d'une force capable de tirer la cellule, un peu à la manière d'un tapis roulant. La synthèse locale d'actine, directement à proximité des sites de polymérisation, permet probablement de faciliter le processus [5-7]. Comme la localisation de l'ARNm de l'actine $\beta$ est, dans les fibroblastes, dépendante des microfilaments d'actine eux-mêmes [9], I'ARNm de l'actine $\beta$ est automatiquement transporté à proximité de la leading edge et une boucle de rétrocontrôle positive se met alors en place. Cette boucle pourrait ainsi faciliter un mouvement continu dans la même direction.

\section{Mécanismes généraux de localisation des ARNm}

La localisation des ARN dépend de déterminants de séquence présents sur ces ARN [10]. Les élements importants de ces déterminants peuvent être soit des structures secondaires et tertiaires plus ou moins complexes, soit des régions simple brin dont la séquence est alors critique. Les facteurs agissant en trans sur ces déterminants comprennent des protéines de reconnaissance de l'ARN, des protéines adaptatrices et, dans la très grande majorité des cas, un ou plusieurs éléments du cytosquelette [9]. Le rôle du cytosquelette est multiple: il peut à la fois servir de support pendant le transport de l'ARN et de point d'ancrage une fois I'ARN arrivé à destination. L'étape de transport permet d'accélérer les mouvements de I'ARN, et elle participe directement à la localisation de l'ARN grâce à sa directionalité. L'étape d'ancrage est essentielle, car c'est elle qui détermine in fine la localisation précise de I'ARN.

Les étapes de transport et d'ancrage peuvent dépendre d'interactions assez directes, le complexe ARN-protéine contactant alors un moteur ou le cytosquelette. À l'opposé, ces étapes peuvent dépendre de structures cellulaires variées comme le fuseau mitotique [11], les centrosomes [12] ou certains domaines membranaires (le réticulum cortical des embryons d'ascidie et de xénope $[1,13]$, ou des compartiments endosomaux [14, 15]). Dans ces cas, I'ARN est alors «opportuniste», et «parasite» en quelque sorte ces structures pour assurer sa localisation.

Cependant, dans de nombreux cas, I'ARN est capable d'assembler une structure spécifiquement destinée à son transport. Ces structures, appelées granules ou, plus récemment, locasomes, ont été mises en évidence dans de nombreux systèmes: la levure [16], les embryons et cellules somatiques de drosophile [17], ou encore les cellules somatiques de vertébrés, principalement les neurones $[18,19]$. L'assemblage d'une structure dévolue au transport de I'ARN procure plusieurs avantages. D'une part, l'efficacité du transport est augmentée, puisque pour chaque événement de transport, c'est plusieurs molécules d'ARN qui vont être déplacées. D'autre part, l'assemblage d'une telle structure permet le cotransport de protéines importantes pour le devenir et la fonction de l'ARNm, ainsi qu'un contrôle groupé de ces molécules. Ainsi, dans les oligodendrocytes et les neurones, les granules d'ARNm contiennent de nombreuses protéines importantes pour la traduction de I'ARNm, comme des ribosomes, des aminoacyl ARNt synthétases et des facteurs de traduction [20].

\section{Mobilité des ARNm dans le cytoplasme}

\section{ARNm non localisés}

Comme la localisation des ARNm dans des régions précises de la cellule est très souvent liée à des propriétés particulières de transport et d'ancrage, il est intéressant de rappeler brièvement ce que l'on sait sur ces processus dans le cas d'ARN non localisés. Par des expériences de fractionnement et de localisation par immunofluorescence ou microscopie électronique, on a pu montrer que la majorité des ARNm sont liés au cytosquelette dans les fibroblastes et les neurones [21-23]. Par ailleurs, l'utilisation de traceurs fluorescents comme les dextrans, non spécifiques mais de taille similaire à un ARNm, a permis de montrer que le cytoplasme est un milieu hétérogène dont la viscosité est proche de celle de l'eau [24]. L'analyse de particules lipidiques naturellement présentes dans le cytoplasme a montré que celles-ci peuvent diffuser, mais de manière non linéaire, avec un coefficient apparent de diffusion diminuant constamment lorsque des intervalles de temps de plus en plus longs sont considérés [25]. Ce type de comportement peut être dû soit à l'hétérogénéité du milieu (par exemple si celui-ci est dynamique et comporte des mailles de taille variable), soit à des associations transitoires avec des éléments stables de la cellule.

Récemment, il a été possible d'analyser directement la mobilité de molécules individuelles d'ARNm dans les cellules vivantes [26], grâce à une technique qui utilise des fusions de la GFP (green fluorescent protein) avec la protéine coat du bactériophage MS2. Cette protéine se lie avec une affinité élevée à une petite tige-boucle 
particulière ( $\mathrm{ms} 2$ ), et des répétitions de ces sites ms2 sont insérées dans la région 3' non-traduite des ARN rapporteurs que l'on veut observer $([16,26]$; Figure 1A). L'observation de molécules uniques d'un ARNm codant pour lacZ, dans les cellules Cos de singe, révèle qu'environ un tiers ( $35 \%$ ) des ARNm sont immobiles, probablement attachés au cytosquelette. Le reste des molécules a, dans sa très grande majorité, un comportement diffusif, soit restreint $(40 \%)$, de même type que celui des gouttelettes lipidiques, soit, plus rarement, pas ou peu restreint $(20 \%)$. Finalement, une minorité d'ARNm ( $<5 \%)$ montre des mouvements motorisés sur des microtubules (Figure IB). Compte tenu de

$\mathbf{A}$

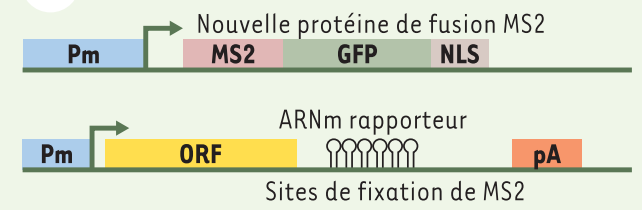

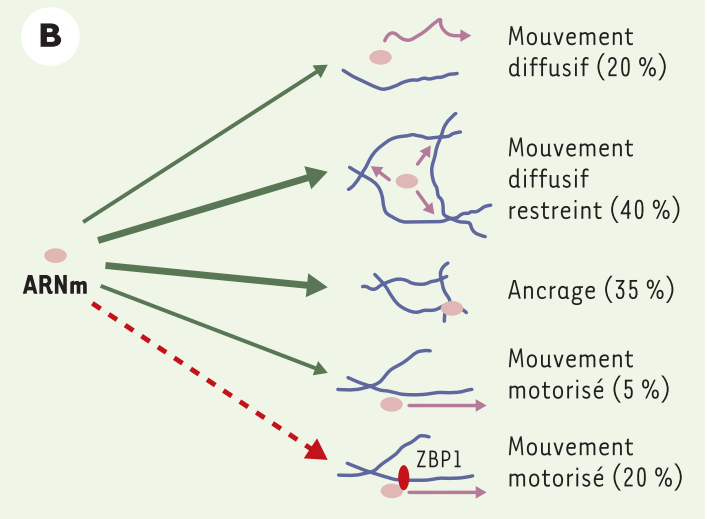

Figure 1. Mouvements des ARNm non localisés et de l'ARNm de l'actine $\beta$. A. Stratégie utilisée pour visualiser des molécules uniques d'ARNm dans les cellules vivantes. Les cellules sont transfectées avec deux plasmides ( $\mathrm{Pm})$, le premier codant pour une protéine de fusion entre la GFP (green fluorescent protein) et la protéine coat du bactériophage MS2, le second pour un ARN rapporteur contenant des répétitions de sites de liaisons à MS2 dans sa région 3' non-traduite. La protéine de fusion (GFP) contient également un signal de localisation nucléaire (NLS), ce qui permet de diriger vers le noyau la protéine non liée à I'ARN. Ainsi, seule la protéine attachée à l'ARN est visible dans le cytoplasme. ORF: open reading frame. B. Comportement des ARNm non localisés dans le cytoplasme de cellules Cos de singe. Quatre comportements sont possibles, suivant l'environnement de la molécule d'ARN. La liaison de la protéine ZBPI (zipcode binding protein 1) (en rouge) sur la séquence zipcode de l'ARNm de l'actine (en rose) entraîne une augmentation de la fréquence et de la longueur de ses mouvements motorisés. la faible diffusion des ARNm dans le cytoplasme, ces mouvements pourraient servir à répartir plus efficacement les ARNm dans la totalité de l'espace cytoplasmique. Une telle fonction serait particulièrement importante dans des types cellulaires comme les neurones, où les distances entre le noyau et la périphérie cellulaire peuvent être très importantes.

\section{ARNm de l'actine $\beta$}

La mobilité des ARNm de l'actine $\beta$ a été analysée grâce à la même technique et dans le même système cellulaire [26] (Figure 1B). Les molécules uniques d'ARNm de l'actine $\beta$ montrent les mêmes types de comportement que les ARNm non localisés, avec cependant deux différences majeures: la fréquence des mouvements motorisés est environ 4 fois plus élevée que celle des ARNm non localisés ( $20 \%$ contre $5 \%$ ), et ces mouvements transportent I'ARNm sur des distances plus grandes ( $7 \mu \mathrm{m}$ en moyenne contre 2,5). Ces observations montrent que si les ARN localisés et non localisés ont des catégories similaires de mouvement (pseudodiffusifs, motorisés, ancrage), les ARN localisés les ont adaptés sur le plan quantitatif afin d'obtenir une distribution intracellulaire précise.

\section{Fondements moléculaires du transport des ARNm de l'actine $\beta$}

Les mécanismes de localisation de l'ARNm de l'actine $\beta$ ont été étudiés en détail. Dans les fibroblastes, I'ARNm est intimement lié au cytosquelette d'actine et dépend de ce réseau pour sa localisation [9]. En revanche, dans les neurones, cet ARNm est préférentiellement associé aux microtubules, et les requiert pour se localiser [27]. La distribution périphérique de I'ARNm de l'actine $\beta$ dépend d'une séquence de 54 nt de sa région 3' non-traduite, le zipcode. À l'heure actuelle, cinq protéines se liant au zipcode ont été isolées et identifiées [28]. Certaines de ces protéines, comme ZBP1 et ZBP2 (zipcode binding protein), sont de la famille des hnRNP (heterogeneous nuclear ribonucleoprotein). ZBPl, conservée au cours de l'évolution, est par exemple nécessaire dans le xénope pour le transport de l'ARNm Vgl au pôle végétatif de l'œuf [29, 30].

Dans des lignées déficientes pour ZBPI, I'ARNm de l'actine $\beta$ est délocalisé dans le cytoplasme périnucléaire. Cependant, l'expression forcée de ZBPl induit la relocalisation de l'ARNm endogène dans ces cellules [31]. De plus, lorsque la localisation de ZBPI est dirigée artificiellement vers un nouveau compartiment cellulaire, par exemple en fusionnant ZBPI à une protéine membranaire, I'ARNm suit la distribution de ZBPI [31]. Ces 
données démontrent que ZBPl est directement impliquée dans la localisation de l'ARNm de l'actine $\beta$.

Dans les fibroblastes, ZBPI est localisée principalement dans le cytoplasme où elle se colocalise, avec I'ARNm de l'actine $\beta$, à proximité des zones riches en actine. De manière surprenante, ZPBl se lie à la fois aux microfilaments et aux microtubules, et est capable de former des granules qui montrent des mouvements motorisés sur les deux types de structures [31]. ZBPI peut ainsi utiliser les deux types de supports pour transporter I'ARNm de l'actine $\beta$, avec cependant des préférences cellulaires: les microfilaments dans les fibroblastes [31], et les microtubules dans les neurones [32]. Le bloquage de la localisation de l'ARNm de l'actine $\beta$ à l'aide d'oligonucléotides antisens du zipcode entraîne la délocalisation de ZBPl, ce qui suggère que l'interaction ARN-protéine est essentielle au transport du complexe ribonucléoprotéique.

Les événements nucléaires sont déterminants dans la localisation cytoplasmique de l'ARN de l'actine $\beta$. D'une part, une étude dynamique de la localisation de ZBPI montre que celle-ci est présente en très faible concentration dans le noyau, et y séjourne brièvement pour s'associer à I'ARN immature durant la transcription [31]. En accord avec ces données, ZBPl possède des signaux d'import et d'export nucléaire [28]. D'aure part, ZBP2, la deuxième protéine qui se lie au zipcode, est localisée principalement dans le noyau. ZBP2 affecte aussi la localisation de l'ARNm de l'actine $\beta$ [33], et les observations actuelles suggèrent que ZBPl et 2 se lient séquentiellement à l'ARN, plutôt que simultanément (Figure 2).

\section{Localisation des ARNm de l'actine $\beta$ dans les neurones}

L'importance de l'actine lors du développement neuronal et de la maturation de la synapse suggère un rôle important pour la localisation de cet ARNm dans les neurones. Effectivement, une étude récente a démontré l'importance de la localisation de l'ARNm de l'actine $\beta$ dans les cônes de croissance des neurones en développement [32]. L'ARNm de l'actine $\beta$ et ZBPl sont tous deux présents dans la zone centrale des cônes de croissance. Des facteurs de croissance comme la neurotrophine-3 (NT-3) induisent l'avancée du cône de croissance, par un mouvement qui dépend de la polymérisation rapide de l'actine dans cette région. La stimulation avec des facteurs de croissance augmente aussi la quantité totale d'ARNm de l'actine $\beta$ présente dans les dendrites et les axones en croissance. La présence d'oligonucléotides antisens spécifiques du zipcode durant la stimulation par NT-3 entraîne une délocalisation de l'ARNm de l'actine $\beta$ et de ZBPl vers le corps cellulaire [32], et des mouvements réduits ou rétrogrades des cônes de croissance. Une diminution de la concentration de l'actine dans les cônes de croissance est aussi observée, indiquant que la synthèse locale de la protéine est essentielle au processus normal de croissance.

La localisation des ARNm au niveau postsynaptique et la synthèse locale de protéine sont l'objet de nombreux travaux étudiant les mécanismes sous-jacents de la plasticité synaptique [34]. Outre son rôle dans la croissance neuronale, l'actine $\beta$ joue un rôle important dans la maturation des épines dendritiques, suggérant qu'elle intervient dans la plasticité synaptique. La stimulation excitatrice par le glutamate affecte la dynamique de l'actine dans les épines dendritiques. Parallèlement, l'actine joue un rôle important dans la réorganisation, ou clustering, des récepteurs glutamatergiques. L'ARNm de l'actine $\beta$ et $Z B P I$ sont présents dans les régions postsynaptiques, dont les épines dendritiques, et une souspopulation de ZBPl est colocalisée avec l'ARNm [35]. L'induction d'une activité neuronale entraîne une relocalisation de l'ARNm de l'actine $\beta$ en parallèle avec un changement de distribution de ZPBI [35]: après dépolarisation neuronale par $\mathrm{du} \mathrm{KCl}$, une augmentation rapide de la protéine ZBPI et de l'ARNm de l'actine $\beta$ est observée dans les régions proximales, médiales et distales des dendrites, ainsi que dans les épines dendritiques, et on

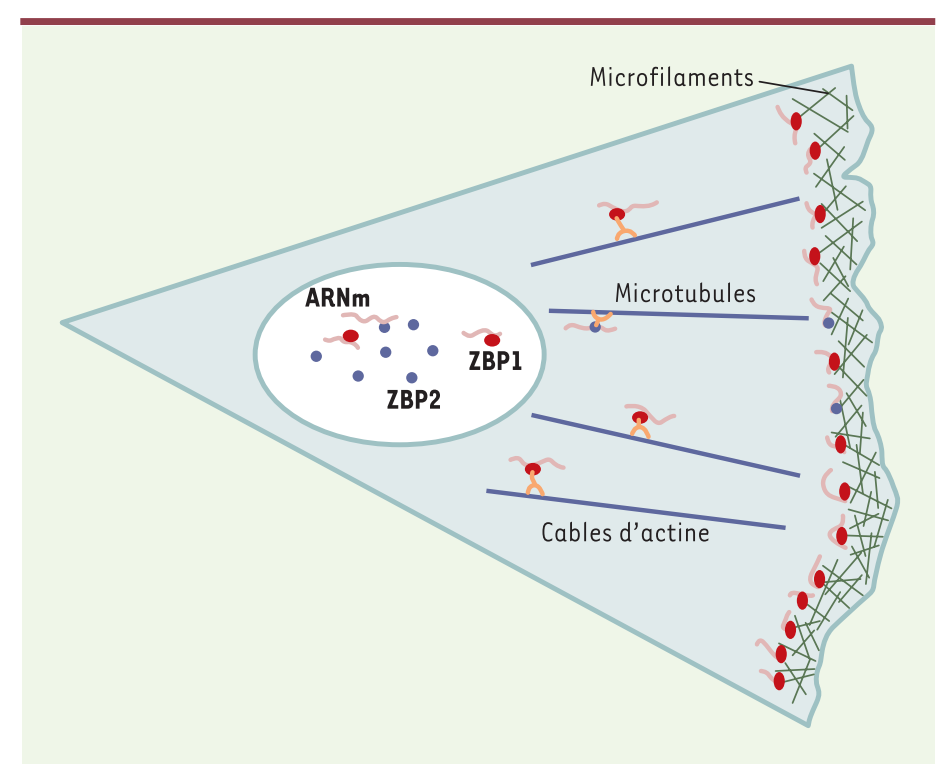

Figure 2. Mécanisme de localisation de l'ARNm de l'actine $\beta$ dans les fibroblastes. L'ARNm l'actine $\beta$ se lie aux protéines ZBP1 et ZBP2 (zipcode binding protein 1 et 2 ) dans le noyau, peut-être de manière séquentielle. Dans le cytoplasme, ZBPI et 2 forment des granules capables de se déplacer sur les microtubules et les filaments d'actine à l'aide de moteurs moléculaires. Une fois arrivé à proximité des zones riches en actine de la périphérie, I'ARNm s'accroche sur ces filaments et y est traduit. 
note une augmentation de la colocalisation de l'ARN et de la protéine ZBPI dans les granules. Cette induction est bloquée par la préincubation des cellules avec un inhibiteur des récepteurs au NMDA ( $\mathrm{N}$-méthyl-d-aspartate), indiquant que cette induction est assurée au moins en partie par une stimulation glutamatergique.

\section{Conclusions}

L'étude de la localisation de l'ARNm de l'actine $\beta$ s'est révélée fascinante et pleine de surprises. L'une des dernières observations réalisées concerne une maladie génétique neuromusculaire très fréquente chez I'homme, la SMA (spinal muscular atrophy, amyotrophie spinale), qui pourrait trouver son origine dans un défaut de transport de cet ARNm [36]. II ne fait nul doute que l'avenir réserve encore de belles découvertes. $\diamond$

\section{SUMMARY}

\section{Cell polarity and actin mRNA localization}

In many species, intracellular mRNA localization is linked to cell polarity. In many cases however, mRNAs become localized as a result of a pre-existing cell-polarity, and they do not modify it. Remarkably, in the case $\beta$ actin mRNA in vertebrate, it has been shown that the transport and localization of this RNA is required for the establishment and maintenance of cell polarity. This occurs in fibroblasts, but, very interestingly, in immature neurons as well. This review will describe the functions and mechanisms of actin mRNA localization. $\diamond$

\section{RÉFÉRENCES}

1. Prodon F, Prulière G, Chenevert J, Sardet C. Établissement et expression des axes embryonnaires: comparaison entre différents organismes modèles. Med Sci (Paris) 2004; 20 : 526-38.

2. Bouget F, Gerttula $S$, Shaw $S$, Quatrano R. Localization of actin mRNA during the establishment of cell polarity and early cell divisions in Fucus embryos. Plant Cell 1996; 8: 189-201.

3. Lawrence J, Singer R. Intracellular localization of messenger RNAs for cytoskeletal proteins. Cell 1986; 45: 407-15.

4. Kislauskis $\varepsilon$, Li Z, Singer R, Taneja K. Isoform-specific 3'-untranslated sequences sort alpha-cardiac and beta-cytoplasmic actin messenger RNAs to different cytoplasmic compartments. J Cell Biol 1993; 123: 165-72.

5. Kislauskis $\varepsilon$, Zhu X, Singer R. Sequences responsible for intracellular localization of beta-actin messenger RNA also affect cell phenotype. J Cell Biol 1994; 127: 441-51.

6. Kislauskis $\varepsilon$, Zhu X, Singer R. Beta-actin messenger RNA localization and protein synthesis augment cell motility. J Cell Biol 1997; 136: 1263-70.

7. Shestakova $\varepsilon$, Singer R, Condeelis J. The physiological significance of beta-actin mRNA localization in determining cell polarity and directional motility. Proc Natl Acad Sci USA 2001; 98: 7045-50.

8. Shestakova $\varepsilon$, Wyckoff J, Jones J, et al. Correlation of beta-actin messenger RNA localization with metastatic potential in rat adenocarcinoma cell lines. Cancer Res 1999; 59 : 1202-5.

9. Sundell C, Singer R. Requirement of microfilaments in sorting of actin messenger RNA. Science 1991; 253: 1275-7.

10. Bashirullah A, Cooperstock RL, Lipshitz HD. RNA localization in development. Annu Rev Biochem 1998; 67: 335-94.
11. Groisman I, Huang Y, Mendez R, et al. CPEB, maskin, and cyclin Bl mRNA at the mitotic apparatus: Implications for local translational control of cell division. Cell 2000; 103: 435-47.

12. Lambert J, Nagy L. Asymmetric inheritance of centrosomally localized mRNAs during embryonic cleavages. Nature 2002; 420: 682-6.

13. Deshler J, Highett M, Schnapp B. Localization of Xenopus Vgl mRNA by Vera protein and the endoplasmic reticulum. Science 1997; 276: 1128-31.

14. Jankovics F, Sinka R, Erdelyi M. An interaction type of genetic screen reveals a role of the Rabll gene in oskar mRNA localization in the developing Drosophila melanogaster oocyte. Genetics 2001; 158: 1177-88.

15. Basyuk E, Galli T, Mougel M, et al. Retroviral genomic RNAs are transported to the plasma membrane by endosomal vesicles. Dev Cell 2003; 5 : 161-74.

16. Bertrand $\varepsilon$, Chartrand $P$, Schaefer M, et al. Localization of ASHI mRNA particles in living yeast. Mol Cell 1998; 2: 437-45.

17. Li P, Yang X, Wasser $M$, et al. Inscuteable and Staufen mediate asymmetric localization and segregation of prospero RNA during Drosophila neuroblast cell divisions. Cell 1997; 90: 437-47.

18. Knowles R, Sabry J, Martone M, et al. Translocation of RNA granules in living neurons. J Neurosci 1996; 16: 7812-20.

19. Ainger K, Avossa D, Morgan F, et al. Transport and localization of exogenous myelin basic protein mRNA microinjected into oligodendrocytes. J Cell Biol 1993; 123: 431-41.

20. Barbarese $\varepsilon$, Koppel D, Deutscher $M$, et al. Protein translation components are colocalized in granules in oligodendrocytes. J Cell Sci 1995; 108: 2781-90.

21. Taneja KL, Lifshitz LM, Fay FS, Singer RH. Poly(A) RNA codistribution with microfilaments: Evaluation by in situ hybridization and quantitative digital imaging microscopy. J Cell Biol 1992; 119: 1245-60.

22. Bassell G, Powers C, Taneja K, Singer R. Single mRNAs visualized by ultrastructural in situ hybridization are principally localized at actin filament intersections in fibroblasts. J Cell Biol 1994; 126: 863-76.

23. Bassell G, Singer R, Kosik K. Association of poly (A) mRNA with microtubules in cultured neurons. Neuron 1994; 12: 571-82.

24. Luby-Phelps K, Castle P, Taylor D, Lanni F. Hindered diffusion of inert tracer particles in the cytoplasm of mouse $3 T 3$ cells. Proc Natl Acad Sci USA 1987; $84:$ 4910-3.

25. Yamada S, Wirtz D, Kuo SC. Mechanics of living cells measured by laser tracking microrheology. Biophys J 2000; 78: 1736-47.

26. Fusco D, Accornero N, Lavoie B, et al. Single mRNA molecules demonstrate probabilistic movement in living Mammalian cells. Curr Biol 2003; 13: 161-7.

27. Bassell G, Zhang H, Byrd A, et al. Sorting of beta-actin mRNA and protein to neurites and growth cones in culture. J Neurosci 1998; 18: 251-65.

28. Ross A, Oleynikov $y$, Kislauskis $\varepsilon$, et al. Characterization of a beta-actin mRNA zipcode-binding protein. Mol Cell Biol 1997; 17: 2158-65.

29. Havin L, Git A, Elisha Z, et al. RNA-binding protein conserved in both microtubule- and microfilament-based RNA localization. Genes Dev 1998; 12: 1593-8.

30. Deshler J, Highett M, Abramson T, Schnapp B. A highly conserved RNAbinding protein for cytoplasmic mRNA localization in vertebrates. Curr Biol 1998; 8: 489-96.

31. Oleynikov $Y$, Singer R. Real-time visualization of ZBPI association with beta-actin mRNA during transcription and localization. Curr Biol 2003; 13: 199-207.

32. Zhang $H$, Eom $T$, Oleynikov $Y$, et al. Neurotrophin-induced transport of a beta-actin mRNP complex increases beta-actin levels and stimulates growth cone motility. Neuron 2001; 31: 261-75.

33. Gu W, Pan F, Zhang H, et al. A predominantly nuclear protein affecting cytoplasmic localization of beta-actin mRNA in fibroblasts and neurons. J Cell Biol 2002; 156: 41-51.

34. Richter J, Lorenz L. Selective translation of mRNAs at synapses. Curr Opin Neurobiol 2002; 12 : 300-4.

35. Tiruchinapalli D, Oleynikov $Y$, Kelic $S$, et al. Activity-dependent trafficking and dynamic localization of zipcode binding protein 1 and beta-actin mRNA in dendrites and spines of hippocampal neurons. J Neurosci 2003; 23: 3251-61.

36. Rossoll W, Jablonka S, Andreassi C, et al. Smn, the spinal muscular atrophy-determining gene product, modulates axon growth and localization of beta-actin mRNA in growth cones of motoneurons. J Cell Biol 2003; 163: 801-12.

\section{TIRÉS À PART}

B. Lavoie 